\title{
Audit of start of anticoagulation treatment in inpatients
}

\author{
G Tan, H Cohen, F Taylor, J Gabbay
}

\begin{abstract}
Aims: To develop a method for evaluating the start of anticoagulation treatment in inpatients.
\end{abstract}

Methods: One hundred case notes were audited using a proforma based on local guidelines in accordance with British Society for Haematology recommendations.

Results: Confirmatory investigations were done in $93 \%$ and $79 \%$ of patients with symptomatic deep venous thrombosis and pulmonary embolism, respectively. Identification of patients' risk factors for anticoagulation by history taking and laboratory tests was often inadequate: baseline coagulation screen, platelet count, liver function and renal function tests were done in $52 \%, 95 \%, 70 \%$ and $87 \%$ of cases, respectively. There was a tendency to undertreat patients: $33 \%$ of the activated partial thromboplastin times (APTT) and $58 \%$ of the International Normalised Ratios (INR) were subtherapeutic. The heparin-warfarin crossover period was particularly problematic: $37 \%$ stopped heparin without an INR that day, or had an INR of less than 2. Microscopic haematuria was monitored occasionally. Of the 62 patients continuing anticoagulation, $72 \%$ were discharged with the final INR in the therapeutic range. At discharge, only $74 \%$ of patients had documented appointments for the anticoagulant Clinic, the period between discharge and appointment ranging from 0 to 12 days. Of the 25 cases with an appointment exceeding four days after discharge, only six $(24 \%)$ had arrangements for an interim INR check. Conclusions: The experience allowed the proforma to become streamlined to a more practical, reliable, and valid tool for use elsewhere. Findings will be fed back to the hospital staff to promote practice improvements before closing the audit loop by re-evaluating practice. Further studies are in progress to identify barriers experienced by doctors in implementing the guidelines and problems in the process of referral to the anticoagulant clinic.

(F Clin Pathol 1993;46:67-71)

Anticoagulants are commonly used in cardiology and in the prevention and treatment of venous thrombosis and pulmonary embolism. In 1982, a quarter of a million people in the
United Kingdom were estimated to be receiving oral anticoagulants each year; since then the numbers have increased. ${ }^{2}$ Not only does the quality of patient care depend on effective anticoagulation, but if clinical research on such patients cannot rely on effective anticoagulant control, the research findings may be misleading. Fortunately the technical control of anticoagulation is now good, achieved mainly through use of the International Normalised Ratio (INR) system and careful standardisation of thromboplastins.

The study site is a 580 bed acute general hospital, with anticoagulation services comparable with that of most district general hospitals. Inpatients may start anticoagulation under the care of any physician, surgeon, or gynaecologist. Hence, although there are guidelines on anticoagulant treatment from the hospital haematologist, in reality there is wide variation in anticoagulation management. An anticoagulant clinic supervised by a consultant haematologist is available for monitoring and advising outpatients. Referral of patients to the anticoagulant clinic occurs either before discharge from a ward, direct from another outpatient clinic, or on discharge from another hospital where anticoagulation was started but would not be followed up.

The British Society for Haematology issued guidelines on the use and monitoring of heparin treatment in $1987^{3}$ and revised the guidelines on oral anticoagulation in $1990 .^{2} \mathrm{At}$ the study hospital, guidelines on the inpatient management of anticoagulant treatment for deep venous thrombosis and pulmonary embolism were drawn up in accordance with those issued by the British Society for Haematology by a consultant haematologist after discussion at the hospital physicians' audit meetings. ${ }^{4}$ These have been circulated to all junior medical staff since 1989, and include specific recommendations for confirmatory investigations, heparin and warfarin initiation, timing and frequency of tests for anticoagulation control, enquiry of risk factors such as interactive medication, duration of anticoagulant courses, and referral to the anticoagulant clinic.

The aim of this study was to develop a method for evaluating the management of inpatients starting heparin. Specific objectives were to design an audit proforma for use on inpatient medical case notes, to pilot its applicability and usefulness at an acute general hospital and to conduct an audit of the practice at this hospital. 


\section{Methods}

An audit proforma was designed, based on the hospital guidelines. The questions were grouped under the key headings of patient particulars, admission/discharge information, initial decision to anticoagulate, evaluation of risk factors for anticoagulation, initiation of anticoagulant treatment, management of anticoagulant treatment, documentation of decisions, discharge communication and information to the patient. A pilot study of both proforma and method was conducted on 20 cases. This included a reliability study of the proforma questions by crosschecking two independent auditors (the research assistant and medically trained research fellow) using $\kappa$ analyses. This test is useful for comparing agreement between two observers classifying nominal categories, taking into account the agreement that might be expected by chance alone. We accepted questions as reliable when the $\kappa$ score achieved a significance level of $\mathrm{p}<0.01$.

At this hospital the tests for anticoagulant control are the activated partial thromboplastin time (APTT) for heparin and the International Normalised Ratio (INR) for warfarin. The normal range for the APTT is 30 to 40 seconds and the recommended range for control of therapeutic intravenous heparin is $\mathbf{5 0}$ to 80 seconds. A common therapeutic range for warfarin (of INR between 2.0 to 4.5 ) is adopted during the induction phase of anticoagulation, and the INR ranges advised by the British Society for Haematology for different conditions applied after the patient has stabilised (for example, INR of $2 \cdot 0-3.0$ for treatment of deep vein thrombosis or in atrial fibrillation; INR of 3.0-4.5 for recurrent deep vein thrombosis or mechanical prosthetic heart valves). $^{2}$

Patients were first identified from the haematology laboratory worksheet if they had had either an isolated APTT or an APTT simultaneous with an INR. They were then entered as cases if it was confirmed when crosschecked with the medical notes that the patient had been treated with heparin. Patients who had heparin prophylactically, including patients already receiving longterm warfarin, were excluded from the study. Inpatient medical notes were retrieved retrospectively for all identified as cases from April to July 1991, and prospectively for such patients from August to October 1991. Retrospective retrieval of inpatient medical notes was achieved by requesting case notes from the medical records department up to five times. Eighty three case notes were requested, for which $79(95 \%)$ were retrieved. Of these, $57(72 \%)$ cases were valid

Table 1 Investigations done to confirm indication to anticoagulate (percentages in parentheses)

\begin{tabular}{llllllll}
\hline & \multicolumn{2}{l}{ Deep vein thrombosis $(n=45)$} & & \multicolumn{2}{c}{ Pulmonary embolism $(n=52)$} \\
\cline { 2 - 3 } & Venogram & Ultrasound & Both & & $Q$ scan & V/Q scan \\
\hline Investigation done & $28(62)$ & $8(18)$ & $6(13)$ & & $41(79)$ & $33(63)$ \\
Delay range (days) & $0-7$ & $0-3$ & $1-11$ & & $0-12$ & $0-6$ \\
Delay median (days) & 1 & 1 & 3 & & 3 & 3 \\
\hline
\end{tabular}

cases and entered into the audit study. Prospective audit of case notes was achieved by obtaining these from medical records, intercepting them when with medical secretaries, on the wards, or at the anticoagulant clinic. All the 63 case notes searched for were successfully retrieved: eight from medical records (six entered the study) and 55 looked for by the research assistant ( 37 entered the study).

\section{Results}

STUDY POPULATION

The 100 audited cases comprised 58 women and 42 men. The age range was 20 to 90 years (median 57 years). The diagnoses for which heparin was given included deep venous thrombosis $(n=40)$, pulmonary embolism $(n=47)$, deep vein thrombosis with pulmonary embolism $(n=5)$, cerebrovascular accident $(n=1)$, systemic embolus other than cerebrovascular accident $(n=4)$, pulmonary embolism with systemic embolus $(n=1)$, cerebrovascular accident with systemic embolus $(n=1)$, and intracardiac thrombus $(n=1)$. A subgroup of 38 patients discontinued anticoagulation, $25(66 \%)$ cases because the confirmatory investigation result was negative, $11(29 \%)$ because the patient died, and two (5\%) because the clinical decision changed on review.

For the 23 cases of deep vein thrombosis who were symptomatic on admission (as opposed to developing the symptoms while already an inpatient for other reasons), the length of stay ranged from four to 17 days (median nine days). For the 18 cases admitted with symptoms or signs of pulmonary embolism, the length of stay ranged from five to 33 days (median nine days).

PRE-ANTICOAGULATION PRACTICE

The guidelines recommend objective investigation to diagnose deep vein thrombosis or pulmonary embolism as soon as possible. Of the 45 cases clinically diagnosed as deep vein thrombosis, 93\% had such investigation, usually a venogram performed within one day (range 0-7 days). Of the six cases which had both venogram and ultrasound scan, two had both tests performed the same day; two had venograms (results positive) before the ultrasound scan; and two had the ultrasound scan (results negative) before the venogram. For the 52 cases diagnosed as pulmonary embolism, $41(79 \%)$ had objective investigation by perfusion scan. Thirty three of these $52(63 \%)$ cases also had ventilation scans, amounting to $80 \%$ of the 41 cases who had perfusion scans. There was a delay between diagnosis and perfusion scan ranging from 0 to 12 days (median three days), and ventilation scan ranging from 0 to six days (median three days)(table 1).

The guidelines state that before starting anticoagulants a check should be made of the patient's current medication (particularly aspirin and non-steroidal anti-inflammatory drugs), age, weight, baseline coagulation screen, platelet count, liver and renal function tests. The drug history was fully documented (complete drug names and doses) in only 69 of 
Table 2 APTT and INR test results (percentages in parentheses)

\begin{tabular}{llll}
\hline & APTT (days 1-10) & INR (days 1-10) & INR (days 4-10) \\
\hline Total tests done & 439 & 272 & 186 \\
Below therapeutic range & $145(33)$ & $158(58)$ & $82(44)$ \\
In therapeutic range & $167(38)$ & $103(38)$ & $93(50)$ \\
Above therapeutic range & $127(29)$ & $11(4)$ & $11(6)$ \\
\hline
\end{tabular}

the 100 cases, and partially documented in 30 cases (the relevant pages were missing in one case). Specific enquiry of aspirin use was made only in 18 cases, and non-steroidal antiinflammatory drugs in five cases. The patient's age and weight were noted in 97 and 70 cases, respectively. Baseline coagulation screen (prothrombin time, APTT, and thrombin time), platelet count, liver function tests and renal function tests were done in 52, 95, 70 and 87 cases, respectively.

\section{INITIATION OF ANTICOAGULATION}

According to the guidelines, all patients starting anticoagulant treatment should receive a bolus dose of intravenous heparin which, with few exceptions, should be 5000 IU. This should be immediately followed by a continuous intravenous infusion of heparin $30000 \mathrm{IU} / 24$ hours, with dose modification for extremes of body weight. Only 29 of the 100 cases received an intravenous bolus dose as recommended. In 10 patients who were maintained on intravenous heparin by continuous infusion, anticoagulation was initiated with a dose of subcutaneous heparin. Of these 39 cases, $34(87 \%)$ received 5000 IU heparin, but one received $3200 \mathrm{IU}$, one $12500 \mathrm{IU}$ and three $10000 \mathrm{IU}$. Because of the difficulty of interpreting entries in the notes, the auditors could not assess the appropriateness of the doses in these latter five patients. For the 75 patients on whom there was sufficient information, it was calculated that heparin was started between two hours to seven days after clinical diagnosis (median six hours). Warfarin was initiated over a period ranging from the day of clinical diagnosis to 25 days later (median two days).

MONITORING ANTICOAGULANT CONTROL

The guidelines recommend that the APTT be

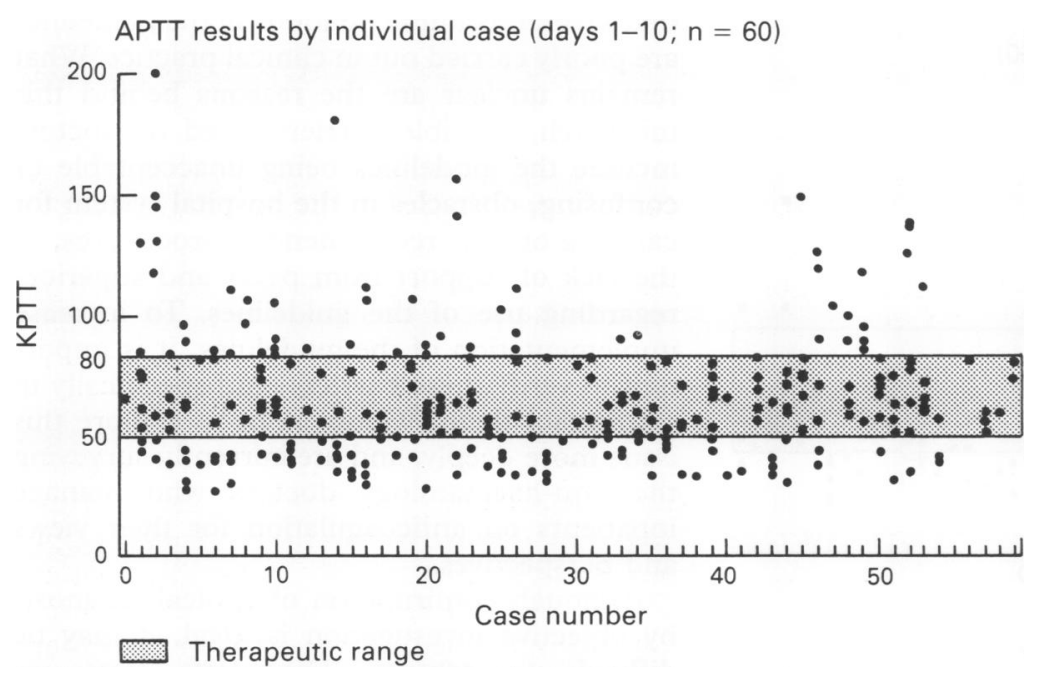

Figure 1 APTT results by individual case (days $1-10 ; n=60$ ). measured four hours after starting the heparin infusion and daily thereafter. More frequent testing may be necessary, detailed in the guidelines, if heparin control is unsatisfactory. Over the first 10 days of anticoagulation, a total of 439 APTT tests were done on 100 cases, $38 \%$ of which were in the therapeutic range ( 50 to 80 seconds), $33 \%$ were below 50 seconds, and $29 \%$ above 80 seconds (table 2, fig 1). The mean APTT calculated for each case who continued anticoagulation ranged from 36 to 109 seconds (mean (SD) 64 (15) seconds).

The hospital guidelines advise doing the first INR check on day 3 of treatment with warfarin. Of the 60 (out of a possible 62) cases where relevant information was not missing or ambiguous, 32 (53\%) cases had an INR done before this (10 cases had INR tests on the first day, 29 cases on the second day). Forty four (73\%) cases did have the INR tested on the third day of warfarin treatment. During the first 10 days of anticoagulation, 272 INR tests were done for the 62 cases continuing anticoagulation. Only $38 \%$ fell in the therapeutic range, $58 \%$ below, and $4 \%$ above (table 2 , fig 2 ). Even if we exclude the results of the first three days before warfarin has achieved full effect, of the 186 INR tests done from days 4 to 10 of warfarin treatment, only $50 \%$ of tests lay in the therapeutic range, $44 \%$ below, and $6 \%$ above (table 2, fig 3 ). Table 2 summarises the APTT and INR results. Omitting one patient who had an INR of more than 8 on one occasion, the remaining mean INR results fell in the range of 1.0 to 6.0 (mean (SD) 2.2 $(0 \cdot 8))$.

It is recommended in the guidelines that heparin be stopped only after the INR equals or exceeds $2 \cdot 0$. Of the 49 cases where relevant information was not missing or ambiguous, 18 (37\%) cases stopped heparin without an INR test that day, or had an INR below $2 \cdot 0$. Another recommendation in the guidelines is that the patient should not be discharged until the INR is stable between $2 \cdot 0$ to 4.5 . We found that although $72 \%$ of the cases were discharged with the final INR in the therapeutic range, $21 \%$ were under $2 \cdot 0$ and $7 \%$ over 4.5 .

Monitoring the side-effects of overanticoagulation is important, and it is recommended in the guidelines that this should include the presence of microscopic haematuria, bruising, and bleeding from venepuncture sites or wounds. Dipstix urinalysis was recorded at least once after starting heparin in only 19 of the $62(31 \%)$ cases continuing anticoagulation. No major bleeds requiring therapeutic intervention were documented. Minor bleeds, frank haematuria which resolved with anticoagulant adjustment, were documented in two cases.

DISCHARGE ARRANGEMENTS

At discharge, an appointment should be made for the earliest anticoagulant clinic, which would normally not exceed seven days, barring bank holidays. Appointments were documented in $74 \%$ of the cases, and in these, the period between discharge and anticoagulant 
INR results by individual case (days $1-10 ; n=60$ )

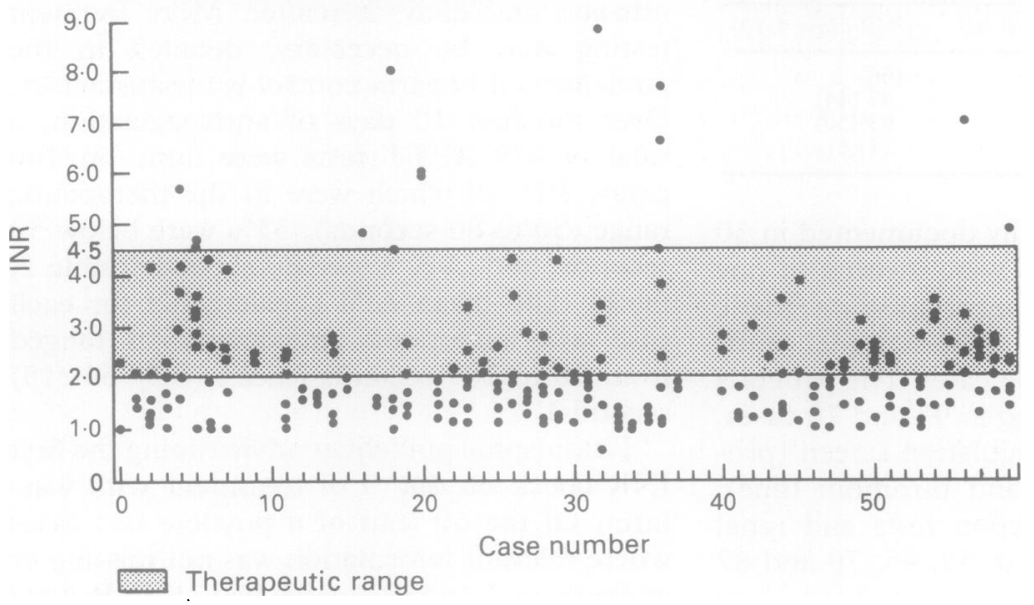

Figure 2 INR results by individual case (days $1-10 ; n=60$ ).

clinic appointment ranged from 0-12 days (median five days). For six (23\%) of the 26 cases with an appointment of four or more days after discharge, a specific arrangement was made to recall the patient to the ward to check the patient's INR during this period.

\section{Discussion}

The purpose of anticoagulant treatment is to prevent the development and complications of thrombosis. Ideally, direct outcome measures of practice would include incidence of thromboembolic events and bleeding episodes (which may indicate under- and overanticoagulation, respectively). In designing the audit proforma, however, we encountered great difficulty in incorporating these as criteria, particularly in extracting this information from the inpatient case notes because of inadequate documentation. Hence we had to use proxy measures, such as APTT and INR ranges for anticoagulation control, and microscopic haematuria for over anticoagulation. It is recognised that microscopic haematuria may be of little clinical importance, but we feel that it may alert the clinician to make a more detailed assessment.

\section{INR results by individual case (days $4-10 ; n=60$ )}

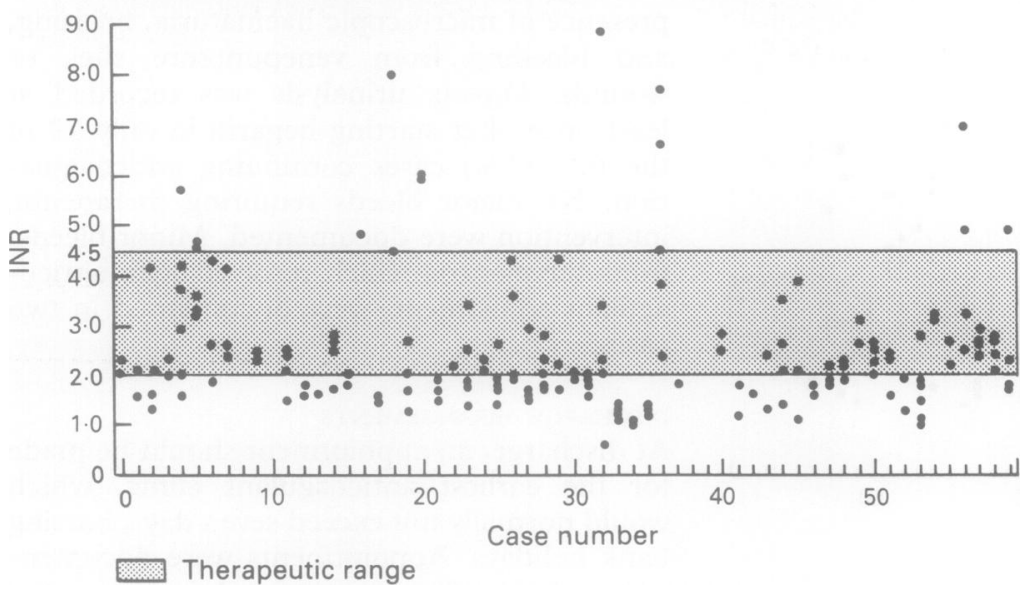

Figure 3 INR results by individual case (days $4-10 ; n=60$ ).
The value of inpatient medical notes in the audit of clinical practice depends on the completeness and accuracy of these as records of relevant events. In the ideal situation, auditors extracting data from case notes would confidently assume that an unrecorded event did not occur. In reality, we found that many events did occur but were poorly recorded, and therefore could not be audited. Examples include putting thrombo-embolic deterrent (TED) stockings on the patient, switching the heparin pump off temporarily, and discussions with the patient. This has been discussed more fully in other studies, ${ }^{4}$ and reinforces the importance of meticulous note-keeping, so that audit using medical records is both feasible and reliable.

Another limitation we encountered in auditing medical notes was the difficulty in interpreting certain documented entries. Hence for many interesting aspects of anticoagulant treatment which we had originally incorporated into the audit proforma, the repeatability among auditors was very poor. For example, a barium meal report "crater in greater curvature" was perceived by the medically trained auditor but not by the research assistant as indicative of a previous peptic ulcer. Other examples of audit criteria we were obliged to reject because of this poor reliability as indicators of quality included pre-anticoagulation consideration of other risk factors such as patient compliance, history of oesophageal varices, history suggestive of a bleeding disorder and heparin hypersensitivity); monitoring of bruising and bleeding from venepuncture sites; and the quality of information given to the patient.

Clinical practice should be periodically evaluated against established guidelines. The guidelines themselves should be regularly reviewed and updated in the light of changes in epidemiology, science, and technology. Guidelines on anticoagulation would be useful only if successfully implemented in day-to-day practice by junior hospital doctors. It is fundamental that the guidelines be distributed widely and explained clearly, and reinforced with sufficient educational impetus. This audit of inpatient notes we conducted has shown that several recommendations of the guidelines are poorly carried out in clinical practice. What remains unclear are the reasons behind this mismatch. Possible barriers faced by doctors include the guidelines being unacceptable or confusing, obstacles in the hospital system for carrying out the recommended procedures, or the lack of support from peers and superiors regarding use of the guidelines. To increase implementation of the guidelines, it is important to verify these barriers, and specifically to counteract them. We are keen to explore this issue more deeply, and are currently surveying the non-haematology doctors who manage inpatients on anticoagulation for their views and perspectives.

Although confirmation of clinical diagnosis by objective investigation is ideal, it may be difficult to achieve. Diagnostic tests are unavailable at nights and weekends, and avail- 
ability may be limited at other times. In addition, there may be unacceptable delays in obtaining test results. The former is the main reason for the unnecessary starting of anticoagulation and calls to question avoidable inconvenience to the patient and waste of hospital resources. On the other hand, heparin is a cheap drug tolerated by most patients when well controlled. Hence the availability of investigations to confirm clinical diagnoses of thromboembolic episodes should be periodically reviewed in the light of changing demand. This should include cost-benefit evaluations of whether increasing radiological facilities and staffing levels would reduce unnecessary inconvenience and costs to patients and hospital. In addition, liaison between ward doctors and radiologists may need to be improved to ensure appropriate tests are obtained promptly.

A lack of appreciation of the pharmacokinetic properties of anticoagulants may account for several of the suboptimal practices. For example, the patient's risk to being anticoagulated is often inadequately assessed in regard to history taking, weight measurement, enquiry of concurrent medication and investigation of baseline coagulation screen, platelet count, liver function and renal function tests. The regimen for initiating anticoagulants using a bolus dose of heparin was inconsistent, and the monitoring of anticoagulation was often illogical (for example, testing the INR before the third day of warfarin treatment) and inadequate (for example, failure to do urinalysis). It may be that the importance of these practices is not adequately appreciated by medical staff, perhaps from inadequate education.

The control of anticoagulation, as indicated by mean APTT and INR values within the therapeutic range, appears reasonable. However, the high percentage of tests, both APTT and INR, falling below therapeutic range suggests a tendency for underanticoagulation by junior hospital doctors, a finding also noted by Doble and Baron. ${ }^{5}$ This may also account for the significant proportion of patients discharged with a final INR below therapeutic range. A particularly important period for anticoagulation control is the crossover of heparin with warfarin. Disappointingly, in a third of the cases heparin was stopped without an INR test that day, or when the INR was below 2.0. Schulman et al advocate stopping heparin only when the INR is within the therapeutic range for two consecutive days, indicating that a stable warfarin effect has been achieved. $^{6}$ Hospital guidelines may need to incorporate a similar specific recommendation.

The handover of patients from the wards to the anticoagulant clinic is a crucial link in the continuity of anticoagulation care. Having committed a patient to anticoagulation while on the ward, it is imperative that follow up as an outpatient be responsibly arranged. Anticoagulant clinic appointments and INR rechecks should be appropriate and referral adequate. Our findings have provided insight to weaknesses which exist at the point of ward discharge, and we plan to develop a more detailed method of auditing this important process of patient referral.

In this study the audit proforma has been successfully applied at the pilot hospital to evaluate inpatient management of anticoagulant treatment using medical notes. The experience has enabled us to streamline the original proforma to a more practical, reliable, and valid tool. We have begun to feed back the results of this study to the hospital staff, and will close the audit loop by re-evaluating inpatient management with the audit proforma after relevant policy changes to improve areas of substandard practice have been implemented. The audit proforma is available on request.

This work is funded by the Research Unit, Royal College of Physicians (UK). We are most grateful to Dr Anthony Hopkins director of the Research Unit, for his invaluable intellectual and practical support.

1 Davies GC, Salzman EW. Cost effectiveness of prophylaxis of venous thrombeombolism. F Roy Soc Med 1981;74 177-80.

2 British Society for Haematology, British Committee for Standards in Haematology Haemostasis and Thrombosis Task Force. Guidelines on oral anticoagulation: Second edition. F Clin Pathol 1990;43:177-83.

3 British Society For Haematology - Report prepared by British Committee for Standards in Haematology HaeBritish Committee for Standards in Haematology Haemostasis and Thrombosis Task Force. Guidelines on the use and monitoring of heparin therapy. In Roberts B, ed. Standard Haematology Practice. Oxford: Blackwell Scien-

4 Gabbay J, McNicol M, Spiby J, Davies SC, Layton AJ. What did medical audit achieve? Lessons from the preliminary evaluation of a year's medical audit. $B M F$ 1990;301 526-9.

5 Doble N, Baron JH. Anticoagulation control with warfarin by junior hospital doctors. $\mathcal{F}$ Roy Soc Med 1987;80:627.

6 Schulman S, Lockner D, Bergstrom K, Blomback M Intensive initial oral anticoagulation and shorter heparin treatment in deep vein thrombosis. Thromb Haemostas 1984;52:276-80. 